\title{
Liquid Structure of Tantalum under Internal Negative Pressure
}

\author{
K. Katagiri $\odot,{ }^{1,2, *}$ N. Ozaki $\odot,{ }^{1,2}$ S. Ohmura,${ }^{3}$ B. Albertazzi, ${ }^{4}$ Y. Hironaka, ${ }^{2,5}$ Y. Inubushi, ${ }^{6,7}$ K. Ishida, ${ }^{1}$ \\ M. Koenig, ${ }^{1,4}$ K. Miyanishi, ${ }^{7}$ H. Nakamura, ${ }^{1}$ M. Nishikino, ${ }^{8}$ T. Okuchi, ${ }^{9}$ T. Sato, ${ }^{10}$ Y. Seto, ${ }^{11}$ \\ K. Shigemori $\odot,{ }^{2}$ K. Sueda, ${ }^{7}$ Y. Tange $\odot,{ }^{6}$ T. Togashi, ${ }^{6,7}$ Y. Umeda $\odot,{ }^{12}$ M. Yabashi $\odot,{ }^{6,7}$ \\ T. Yabuuchi $\oplus^{6,7}$ and R. Kodama ${ }^{1,2}$ \\ ${ }^{1}$ Graduate School of Engineering, Osaka University, Osaka 565-0871, Japan \\ ${ }^{2}$ Institute of Laser Engineering, Osaka University, Osaka 565-0871, Japan \\ ${ }^{3}$ Research Center for Condensed Matter Physics, Department of Environmental and Civil Engineering, \\ Hiroshima Institute of Technology, Hiroshima 731-5193 Japan \\ ${ }^{4}$ LULI, CNRS, CEA, Ecole Polytechnique, UPMC, Université Paris 06: Sorbonne Universites, Institut Polytechnique de Paris, \\ F-91128 Palaiseau cedex, France \\ ${ }^{5}$ Open and Transdisciplinary Research Initiative, OTRI, Osaka University, Osaka 565-0871, Japan \\ ${ }^{6}$ Japan Synchrotron Radiation Research Institute, Hyogo 679-5198, Japan \\ ${ }^{7}$ RIKEN SPring-8 Center, Hyogo 679-5148, Japan \\ ${ }^{8}$ Kansai Photon Science Institute, National Institutes for Quantum and Radiological Science and Technology, \\ Kyoto 619-0215, Japan \\ ${ }^{9}$ Institute for Integrated Radiation and Nuclear Science, Kyoto University, Osaka 590-0494, Japan \\ ${ }^{10}$ Graduate School of Science, Hiroshima University, Hiroshima 739-8526, Japan \\ ${ }^{11}$ Graduate School of Science, Kobe University, Hyogo 657-0013, Japan \\ ${ }^{12}$ Institute for Planetary Materials, Okayama University, Tottori 682-0193, Japan
}

(Received 16 December 2020; revised 9 March 2021; accepted 31 March 2021; published 28 April 2021)

\begin{abstract}
In situ femtosecond x-ray diffraction measurements and ab initio molecular dynamics simulations were performed to study the liquid structure of tantalum shock released from several hundred gigapascals (GPa) on the nanosecond timescale. The results show that the internal negative pressure applied to the liquid tantalum reached -5.6 (0.8) GPa, suggesting the existence of a liquid-gas mixing state due to cavitation. This is the first direct evidence to prove the classical nucleation theory which predicts that liquids with high surface tension can support GPa regime tensile stress.
\end{abstract}

DOI: 10.1103/PhysRevLett.126.175503

The explorations of the phase diagram of most materials are typically focused on their positive pressure part. However, solids and liquids can also be subjected to an internal negative pressure by applying external mechanical tension [1-6]. This lattice stretching can lead to a phase transformation [7] of the material or its fracture [1,2] and it could occur in a wide range of domains including planetary impact events [8] and industrial applications such as laser processing [9].

Cavitation is the boiling of a liquid due to an internal negative pressure rather than raised temperature, and it can cause liquid failure upon shock release of liquids. According to the classical nucleation theory [5], the cavitation pressures of most liquid metals with high surface tensions are predicted to be several gigapascals (GPa) [6], but the experimental investigations to prove the theory has been challenging. Indeed, experimental techniques exist but are restricted to an internal negative megapascal (MPa) range (e.g., Berthelot method [3]).

The advantages of the laser-shock compression techniques are vast as the sample is initially heated and compressed to extremely high temperature and pressure.
Since the drive laser pulse duration is typically up to several nanoseconds, shock pressure remains in the sample only for some nanoseconds. Then the loaded pressure is rapidly released to the ambient pressure or even to internal negative pressure. During the release process, a residual temperature of up to thousands of kelvins would remain in the sample due to the high entropy gained during the compression. Since these compression and release processes typically occur within the timescale of several nanoseconds, an in situ x-ray diffraction (XRD) technique with a high temporal resolution is required to capture the structural change in a material under shock. The recent development of femtosecond pulsed $\mathrm{x}$-ray free electron lasers (XFELs) [10] has enabled such studies to be performed. However, the observations of liquid structures of materials under shock compression by in situ XRD using an XFEL are still challenging $[11,12]$ and no experimental data so far exist on liquids with internal negative pressure. This is even more difficult for materials with high melting temperatures such as tantalum (3270 $\mathrm{K}$ at $0 \mathrm{GPa}$ [13]), where no experimental observation of its liquid structure has been achieved. Here, we report the first experimental observation of the structure 


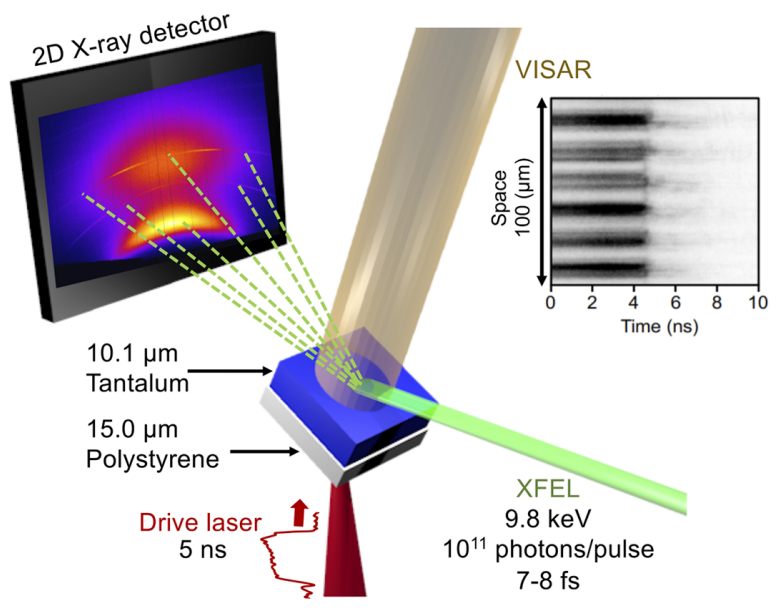

FIG. 1. Experimental configuration and data. Two different types of probes: XFEL and VISAR were used to record XRD pattern and shock breakout timing, respectively [24].

of a liquid (tantalum) sustaining a gigapascal regime negative pressure using high temporal resolution XRD. Besides, the structures of tantalum along its Hugoniot up to 222 (7) GPa have been collected as the research on hightemperature polymorphism and melting of tantalum has received attention in the last few decades [14-21].

The experiments have been performed at SPring-8 Angstrom Compact Free Electron Laser (SACLA) $[22,23]$. Figure 1 illustrates an overview of the experiment. Our targets consist of a $15.0(0.2) \mu \mathrm{m}$ thick polystyrene ablator and a $10.1(0.2) \mu \mathrm{m}$ thick polycrystalline tantalum (Ta) foil of $99.9 \%$ purity (Nilaco Corporation). The thickness of the glue between the polystyrene and $\mathrm{Ta}$ is $1.2(0.3) \mu \mathrm{m}$. The target was shock compressed by irradiating an optical drive laser $(532 \mathrm{~nm}, 5 \mathrm{~ns}$ duration square pulse) [24], and the shock-released state of Ta was observed by irradiating the high intensity $\left(\sim 10^{11}\right.$ photons/pulse) and ultrafast ( $<10 \mathrm{fs}$ ) $\mathrm{x}$-ray pulse of SACLA XFEL at $\sim 1.0 \mathrm{~ns}$ after the shock wave reaches the rear surface of the Ta. The XFEL probe timings were set based on the results of hydrodynamic simulations [24]. The XFEL beam had a photon energy of $9.8 \mathrm{keV}$ and was focused to $30 \times 10(\mathrm{~W} \times \mathrm{H}) \mu \mathrm{m}$. The drive laser and the XFEL beam were incident on the targets at angles of $20^{\circ}$ and $70^{\circ}$, respectively. Diffracted x rays were collected on a twodimensional x-ray detector in reflection geometry. Note that the $\mathrm{x}$-ray attenuation length of $\mathrm{Ta}$ at the fixed angle is $\sim 2 \mu \mathrm{m}$ for $9.8 \mathrm{keV}$, meaning that only the volume near the rear surface of the Ta sample is probed. The drive laser was focused to the spot size of 150-250 $\mu \mathrm{m}$ on target and the beam pattern was smoothed by using a phase plate [24]. The drive laser intensity $\left(<10 \mathrm{TW} / \mathrm{cm}^{2}\right)$ was varied to apply different peak pressures in Ta, resulting in different residual temperatures upon release. The time resolution of the velocity interferometer system for any reflector (VISAR) is $\sim 40 \mathrm{ps}$ and the combined timing jitter of the XFEL and the drive laser is less than 160 ps.
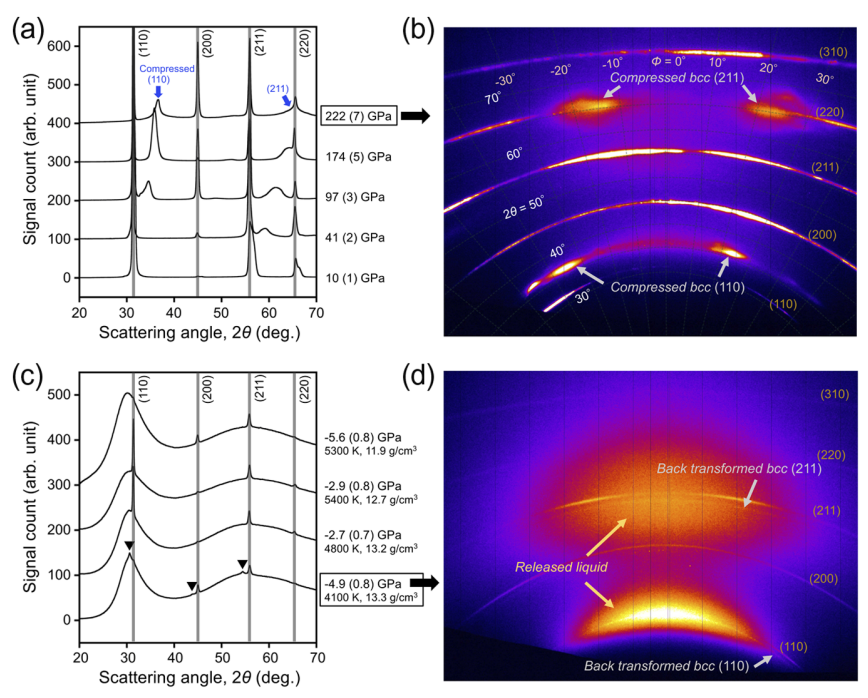

FIG. 2. (a) X-ray diffraction profiles of the shock-compressed Ta with various pressures up to 222 (7) GPa. The peak positions of diffraction from unshocked Ta are indicated by gray lines. The peaks corresponding to the compressed bcc (110) and (211) planes are highlighted by blue arrows for the 222 (7) GPa data. The pressure uncertainty for solid data is estimated from the broadness (FWHM) of the XRD peaks. (b) Recorded XRD image of Ta shock compressed to 222 (7) GPa. Dotted curves and lines indicate constant scattering angle $(2 \theta)$ and azimuth angle $(\varphi)$, respectively. (c) Diffractions patterns of liquid Ta at shock released states with different residual temperatures. Black inverted triangles denote peaks of back-transformed bcc phase with a density of $15.5(0.2) \mathrm{g} / \mathrm{cm}^{3}$. (d) Recorded XRD image of Ta at shock released state of $-4.9(0.8) \mathrm{GPa}$ and 4100 (1100) K. Uncompressed peaks are from Ta at outside of the drive laser focal spot. The signal intensities are lower at higher azimuthal angles, as the absorption of diffracted $\mathrm{x}$ rays by the Ta sample itself becomes more significant at higher azimuthal angles.

The measured XRD data are summarized in Fig. 2. First, the structure of Ta along its Hugoniot (the locus of shock state) up to 222 (7) GPa has been evaluated accurately by in situ XRD diffraction [Fig. 2(a)]. A previous study observed a phase transformation from bcc to hexagonal$\omega$ phase in a recovered sample of Ta initially shocked to $45 \mathrm{GPa}$ [14]. Also, ab initio molecular dynamics (AIMD) simulation predicted the occurrence of solid-solid phase transformations of Ta above $\sim 70 \mathrm{GPa}$ [15]. However, the latest experimental results of shock compressed Ta showed that there are no phase transitions until it finally melts at around $300 \mathrm{GPa}$ [16-19]. The shock pressures noted in Fig. 2(a) are determined from the densities measured by the XRD using the existing Ta Hugoniot [25]. The consistency between the density measured by the XRD and the average shock velocity estimated from the VISAR is checked and described in the Supplemental Material [24]. Our results show that shocked Ta remains the bcc phase at least to 222 (7) GPa, which is consistent with the latest in situ XRD studies $[16,17]$. 
Second, the liquid structure of Ta shock released from peak pressures of 312 (22)-441 (36) GPa were recorded. To determine the shock released state of Ta, we first have to characterize its shock compressed (Hugoniot) state. Hugoniot pressure for the released-liquid analysis is determined from the shock wave transit time using the existing Ta Hugoniot [25]. Polystyrene Hugoniot reported by Barrios et al. [26] was used to estimate the shock wave transit time in the polystyrene ablator. The temperature at the shock-compressed state $T_{H}$ is estimated from the shock pressure $P_{H}$ and volume at the shocked state $V_{H}$, using the Mie-Grüneisen equation of state [27]:

$$
\begin{aligned}
T_{H}(\mu)= & T_{0} \exp \left(\gamma_{0} \mu\right) \\
& -\frac{V_{0} \exp \left(\gamma_{0} \mu\right)}{C_{V}} \int_{0}^{\mu} f(\mu) \exp \left(-\gamma_{0} \mu\right) d \mu
\end{aligned}
$$

where $f(\mu)=-\frac{d P_{H}}{d \mu} \frac{\mu}{2}+\frac{P_{H}(\mu)}{2}, \quad \mu=1-V_{H} / V_{0}$.

Here, $\gamma_{0}=1.53[28]$ is the Grüneisen parameter at ambient and $C_{V}=173.37 \mathrm{~J} / \mathrm{kg} \mathrm{K}$ [29] is the specific heat at constant volume. Subscripts $H$ and 0 denote the Hugoniot state and the ambient condition, respectively.

The density of Ta at its released state is measured from the recorded x-ray scattering pattern [Fig. 2(c)] by using the iterative procedure of Eggert et al. [30,31], which is implemented in a code "LiquidDiffract" [32]. Then, the temperature at the released state $T_{R}$ is determined by using the following equation:

$$
T_{R}=T_{H} \exp \left\{\int_{V_{R}}^{V_{H}}\left(\frac{\gamma}{V}\right) d V\right\} .
$$

Subscript $R$ denotes the released state. $\gamma / V=\gamma_{0} / V_{0}$ is assumed in this work. Heighway et al. recently reported a nonisentropic release behavior in shocked solid Ta due mainly to plastic-work heating [33] but we assume this effect is negligible in our case as our Ta is expected to be melted upon shock compression.

For liquid scattering data analysis, the diffracted $\mathrm{x}$ rays recorded at azimuth angle $(\varphi)$ of $-5 \leqq \varphi \leqq 5$ degrees were analyzed. Here, the shock wave propagation direction is assumed to be along $\varphi=0 \mathrm{deg}$. Coherent scattering intensity $I_{\text {coh }}$ can be obtained from a recorded x-ray intensity profile by removing the effects of absorption, polarization (the XFEL beam of SACLA is polarized horizontally), and Compton scattering. The absorption was corrected by using the absorption coefficient of $\mathrm{Ta}$ which is $\mu / \rho=95.9 \mathrm{~cm}^{2} / \mathrm{g}$ at $9.8 \mathrm{keV}$. The solid peaks are properly removed (masked) to obtain the liquid scattering data. The scattering vector $Q$ and the structure factor $S(Q)$ can be determined by $Q=4 \pi \sin \theta / \lambda$, and $S(Q)=I_{\text {coh }} / N f^{2}$, respectively. Here, $\lambda$ is the wavelength of the probing (a)

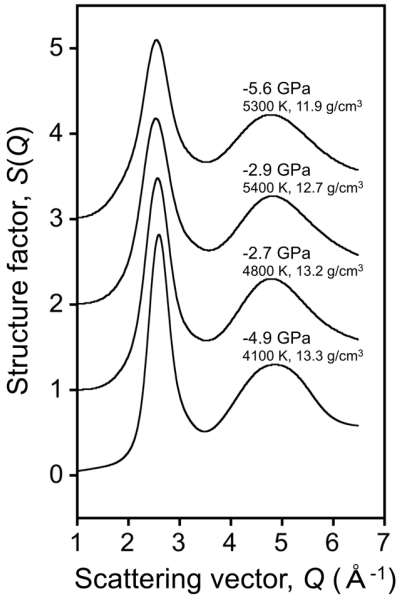

(b)

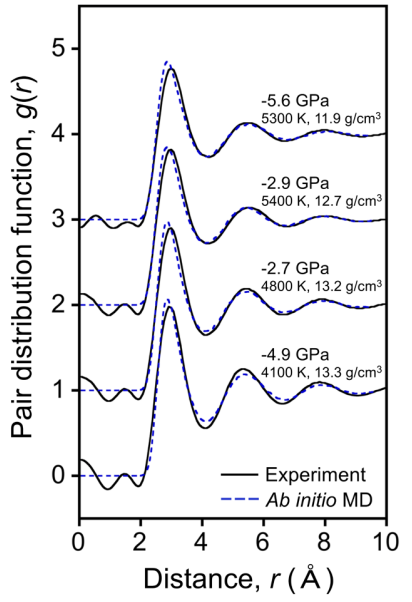

FIG. 3. (a) Structure factors of Ta at the shock-released states. The shown profiles of $Q<1.72$ were extrapolated from those of $Q \geqq 1.72$. (b) Corresponding pair distribution functions of Ta. The experimental results (black solid profiles) are compared with the AIMD simulation results (blue dotted profiles).

$\mathrm{x}$ rays, $N$ is the number of atoms in the system, and $f$ is the atomic scattering factor. The structure factor profiles of $Q<1.72$ shown in Fig. 3(a) were extrapolated from those of $Q \geqq 1.72$, as the diffracted x rays at scattering angles lower than $20^{\circ}$ were significantly absorbed by the Ta sample itself. Then the pair distribution function $g(r)$ can be determined by

$$
g(r)=1+\frac{1}{2 \pi^{2} n r} \int_{0}^{\infty} Q\{S(Q)-1\} \sin (Q r) d Q,
$$

where $n$ and $r$ are the number density and the distance from any given atom, respectively. The Lorch modification function [34] with a $Q$ maximum of $6.50 \AA^{-1}$ was applied to remove the truncation effect during the Fourier transforming process. The $r$-minimum cutoff values for the four shots ranged from 2.28 to $2.35 \AA$.

From the experimental determination of the density and temperature at the shock released state, one can access the pressure of shock-released Ta using AIMD simulation. In our simulations, the atomic forces are obtained from the electronic states calculated using the projector-augmentedwave method [35,36] within the framework of density functional theory. The generalized gradient approximation [37] was used for the exchange correlation energy. The cutoff energies of the plane wave were 17 and 170 Ry for the electronic pseudowave functions and the pseudocharge density, respectively. The energy functional was minimized by using an iterative scheme based on the preconditioned conjugate-gradient method $[38,39]$. The gamma point was used for Brillouin zone sampling. As valence electrons, $6 s, 5 d$, and $6 p$ were used. We used a system of 256 atoms in a cubic supercell under periodic boundary conditions. 
Using the Nòse-Hoover thermostat technique, the equations of motion were solved via an explicit reversible integrator [40] with a time step of $\Delta t=2.4 \mathrm{fs}$. The quantities of interest were obtained by averaging over 7.2 ps. The temperature of the electrons is fixed by the Fermi-Dirac distribution which defines the occupation numbers of bands.

The measured structure factors $S(Q)$ and the pair distribution functions $g(r)$ of Ta shock released from various shock pressures are shown in Fig. 3 along with the AIMD simulation results. A similar trend in $g(r)$ is observed between the AIMD simulations and experimental data. The broadening of the peaks of $g(r)$ becomes larger as the temperature increase. The asymmetry of the first peak observed in the experimental data may be explained by the compatibility with the initial bcc-like structure, as also seen in previously reported molecular dynamics (MD) simulation results [41].

Figure 4 shows the density, pressure, and coordination number at different residual temperatures. The measured densities of $\mathrm{Ta}$ are lower than the densities measured at ambient pressure [green line in Fig. 4(a)] [42], thought to be due to the mechanical tension applied to the liquid Ta. The cavitation pressure $P_{C}$ can be theoretically estimated by

$$
P_{C}=-\left[\frac{16 \pi \sigma^{3}}{3 k_{B} T} \frac{1}{\ln \left(\frac{\Gamma_{0} V \tau}{\ln 2}\right)}\right]^{1 / 2},
$$

where $\sigma, k_{B}, T, V$, and $\tau$ are the liquid surface tension, Boltzmann's constant, the absolute temperature, the volume of the liquid, and the time period, respectively [5]. The parameter $\Gamma_{0}$ is approximated by $\Gamma_{0} \sim 3 k_{B} T / 4 \pi R_{C}^{3} h$, where $h$ is Plank's constant and $R_{C}$ is the critical nucleus radius which is defined as $-2 \sigma / P_{C}$. Paradis et al. measured the surface tension of liquid Ta over the 2970-3400 K interval and their results can be expressed as $\sigma(T)=2.15 \times$ $10^{3}\left( \pm 0.16 \times 10^{3}\right)-(0.21 \pm 0.05)\left(T-T_{m}\right)(\mathrm{mN} / \mathrm{m})$ [43], where $T_{m}$ is the melting temperature of Ta at $0 \mathrm{GPa}$ $(\sim 3270 \mathrm{~K})$. We extrapolated this relationship to $4400 \mathrm{~K}$ to predict the cavitation pressures. We used a constant value of $V=0.0758 \mathrm{~cm}^{3} / \mathrm{g}$ for the entire temperature range as $P_{C}$ is quite insensitive to the $V$. The timescale $\tau$ is assumed to be 1 nanosecond in this work. The predicted cavitation pressure of liquid $\mathrm{Ta}$ as a function of temperature is shown as a blue solid curve in Fig. 4(b). The negative pressures obtained by AIMD are down to -5.6 (0.8) GPa [Fig. 4(b)], close to the cavitation pressures $\left(P_{C}\right)$ estimated by previous MD simulations (red dashed curve) [44]. These results suggest the existence of the cavitation in our shock-released Ta. While the experimentally elucidated pressures agree with the MD simulations, they are less negative than the cavitation pressures predicted by a classical nucleation theory (blue solid curve). We assume that this is a consequence of heterogeneous void nucleation due to the anisotropy of shock release and to slight impurities

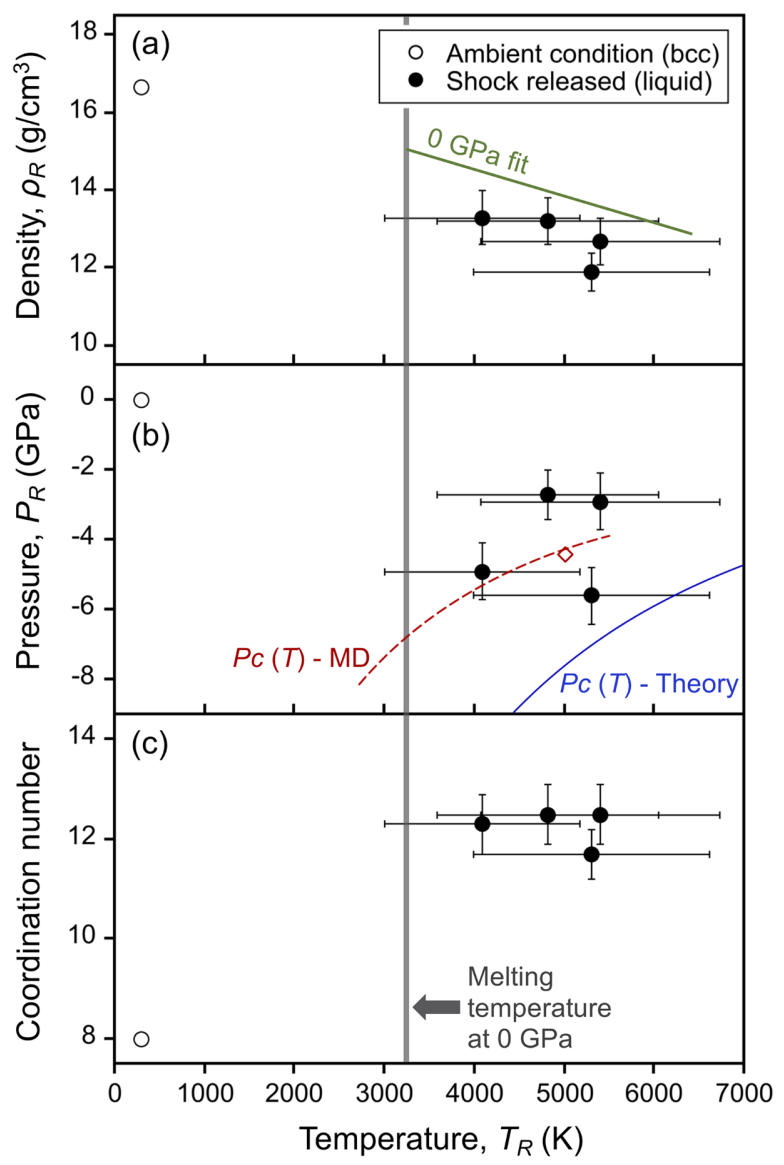

FIG. 4. (a) Density as a function of the temperature at the released state. The uncertainty of the density is estimated based on the uncertainties of the $r$-min cutoff value and the differences in the densities obtained for each iteration process. The gray line indicates the melting temperature of Ta at $0 \mathrm{GPa}(3270 \mathrm{~K})$ and the green solid line shows the density-temperature relationship measured at $0 \mathrm{GPa}$ [42]. (b) Pressures at the released states determined by AIMD simulations using the measured density and temperature as initial simulation parameters. The shown error bars on the pressure are estimated from the fluctuation of the AIMD simulations [24] and the uncertainties on the initial calculation parameters (temperature and density) are not considered. The blue solid curve shows the temperature dependence of the cavitation pressure $P_{C}(T)$ predicted by the classical nucleation theory. The red dashed curve and red open diamond are the cavitation pressures calculated using MD simulations by Hahn et al. [44] and the cavitation pressure of Ta estimated from the experimental results of Ashitkov et al. [47], respectively. At pressures lower than $P_{C}(T)$, liquid Ta is likely to form small-vapor-filled cavities. (c) Coordination number at different residual temperatures.

suspended in the liquid Ta [4]. Such discrepancy between experiment and theory was also seen in water $[45,46]$ and mercury $[4,46]$.

The coordination number $(n)$ can be extracted by $n=\int_{r_{0}}^{r_{\min }} 4 \pi \rho r^{2} g(r) d r$, where $r_{0}$ and $r_{\text {min }}$ are the locations of the left-hand edge and the first minimum to the right of 
the first peak, respectively. The determined coordination numbers are $\sim 12$ [Fig. 4(c)], suggesting that the structure is a simple liquid metal in the wide pressure and temperature ranges studied in this work.

At 4100 (1100) K, around the melting temperature of Ta at $0 \mathrm{GPa}(3270 \mathrm{~K}[13])$, we observed the coexistence of the liquid phase and the back-transformed bcc (solid) phase [Figs. 2(c) and 2(d)]. Cortella et al. used a brightness trace method of $\mathrm{Ta}$ droplets and observed a formation of a metastable phase (the structure is predicted to be A15) at $2930 \mathrm{~K}$ during the supercooling process before the phase transition to a stable bcc phase finally occurs at $2770 \mathrm{~K}$ [48]. However, we did not observe any evidence of a metastable phase during the rapid cooling of the shockrelease process in the present study. This difference can be explained by the fact that the cooling process in our shock experiments is accompanied by a significant and rapid pressure reduction resulting in the pressure at the shockreleased state being much lower than $0 \mathrm{GPa}$, which could be crucial for the formation of the metastable phase.

Presented results revealing liquid Ta can withstand extreme negative pressures down to $-5.6(0.8) \mathrm{GPa}$, are consistent with the classical nucleation theory which suggests that the cavitation pressure of a liquid strongly depends on the material's surface tension. Such understanding of dynamic fracture in liquids is of interest in many scientific and engineering fields and provides new insights into the modeling of liquid failure under rapid shock release.

We thank J. Lintz for his valuable comments on the manuscript. This experiment was performed at BL3 of SACLA with the approval of the Japan Synchrotron Radiation Research Institute (proposals No. 2019B8032 and No. 2019B8057). S. O. thanks the Supercomputer Center, Institute for Solid State Physics, University of Tokyo, for use of their facilities. Simulations were also carried out using the computer facilities at the Research Institute for Information Technology, Kyushu University. This work was supported by grants from MEXT Quantum Leap Flagship Program (MEXT Q-LEAP) Grant No. JPMXS0118067246 and Japan Society for the Promotion of Science (JSPS) KAKENHI (Grants No. 20H00198, No. 19K21866, and No. 16H02246), Genesis Research Institute, Inc. (Konpon-ken, Toyota). B. A. would like to thank CNRS for the support given by a grant for travel expenses (GOtoXFEL). The highpower drive laser installed in SACLA EH5 was developed with the corporation of Hamamatsu Photonics.

*Corresponding author.

kkatagiri@ef.eie.eng.osaka-u.ac.jp

[1] T. Antoun, L. Seaman, D. R. Curran, G. I. Kanel, S. V. Razorenov, and A. V. Utkin, Spall Fracture (Springer, Berlin, 2002).
[2] B. Albertazzi et al., Sci. Adv. 3, e1602705 (2017).

[3] M. Berthelot, Ann. Chim. Phys. 30, 232 (1850).

[4] G. A. Carlson, J. Appl. Phys. 46, 4069 (1975).

[5] J. J. Hoyt and A. A. Potter, Mater. Trans. A 43, 3972 (2012).

[6] E. Erzi and M. Tiryakioḡlu, Mater. Sci. Technol. 35, 1656 (2019).

[7] V. I. Levitas, B. F. Henson, L. B. Smilowitz, and B. W. Asay, J. Phys. Chem. B 110, 10105 (2006).

[8] K. Ernstson, M. R. Rampino, and M. Hiltl, Geology 29, 11 (2001).

[9] K. C. Phillips, H. H. Gandhi, E. Mazur, and S. K. Sundaram, Av. Opt. Photonics 7, 684 (2015).

[10] T. Ishikawa et al., Nat. Photonics 6, 540 (2012).

[11] N. J. Hartley et al., Phys. Rev. Lett. 121, 245501 (2018).

[12] R. Briggs et al., Appl. Phys. Lett. 115, 264101 (2019).

[13] L. Malter and D. B. Langmuir, Phys. Rev. 55, 743 (1939).

[14] L. M. Hsiung and D. H. Lassila, Acta Mater. 48, 4851 (2000).

[15] L. Burakovsky, S. P. Chen, D. L. Preston, A. B. Belonoshko, A. Rosengren, A. S. Mikhaylushkin, S. I. Simak, and J. A. Moriarty, Phys. Rev. Lett. 104, 255702 (2010).

[16] X. Wang et al., Rev. Sci. Instrum. 90, 053901 (2019).

[17] C. E. Wehrenberg et al., Nature (London) 550, 496 (2017).

[18] N. C. Holmes, J. A. Moriarty, G. R. Gathers, and W. J. Nellis, J. Appl. Phys. 66, 2962 (1989).

[19] M. C. Akin, J. H. Nguyen, M. A. Beckwith, R. Chau, W. P. Ambrose, O. V. Fat'yanov, P. D. Asimow, and N. C. Holmes, J. Appl. Phys. 125, 145903 (2019).

[20] A. Dewaele, M. Mezouar, N. Guignot, and P. Loubeyre, Phys. Rev. Lett. 104, 255701 (2010).

[21] D. Errandonea, M. Somayazulu, D. Häusermann, and H. K. Mao, J. Phys. Condens. Matter 15, 7635 (2003).

[22] Y. Inubushi et al., Appl. Sci. 7, 584 (2017).

[23] Y. Inubushi et al., Appl. Sci. 10, 2224 (2020).

[24] See Supplemental Material at http://link.aps.org/ supplemental/10.1103/PhysRevLett.126.175503 for drive laser properties, VISAR timing calibration, shock velocity analysis, consistency between the density measured by the $\mathrm{XRD}$ and the average shock velocity estimated from the VISAR, hydrodynamics simulations, uncertainty estimation of the release pressure, and summarized shock-release data.

[25] A. C. Mitchell and W. J. Nellis, J. Appl. Phys. 52, 3363 (1981).

[26] M. A. Barrios, D. G. Hicks, T. R. Boehly, D. E. Fratanduono, J.H. Eggert, P. M. Celliers, G. W. Collins, and D. D. Meyerhofer, Phys. Plasmas 17, 056307 (2010).

[27] J. M. Walsh and R.H. Christian, Phys. Rev. 97, 1544 (1955).

[28] C. Bercegeay and S. Bernrd, Phys. Rev. B 72, 214101 (2005).

[29] E. N. Hahn and S. J. Fensin, J. Appl. Phys. 125, 215902 (2019).

[30] J. H. Eggert, G. Weck, P. Loubeyre, and M. Mezouar, Phys. Rev. B 65, 174105 (2002).

[31] T. Sato, N. Funamori, and T. Kikegawa, Rev. Sci. Instrum. 81, 043906 (2010).

[32] B. J. Heinen, LiquidDiffract v1.0.0, https://doi.org/10.528/ zenodo.3592511 (2019).

[33] P. G. Heighway et al., Phys. Rev. Lett. 123, 245501 (2019).

[34] E. Lorch, J. Phys. C 2, 229 (1969).

[35] P. E. Blöchl, Phys. Rev. B 50, 17953 (1994). 
[36] G. Kresse and D. Joubert, Phys. Rev. B 59, 1758 (1999).

[37] J. P. Perdew, K. Burke, and M. Ernzerhof, Phys. Rev. Lett. 77, 3865 (1996).

[38] G. Kresse and J. Hafner, Phys. Rev. B 49, 14251 (1994).

[39] F. Shimojo, R. K. Kalia, A. Nakano, and P. Vashishta, Comput. Phys. Commun. 140, 303 (2001).

[40] M. Tuckerman, B. J. Berne, and G. J. Martyna, J. Chem. Phys. 97, 1990 (1992).

[41] N. Jakse, O. Le Bacq, and A. Pasturel, Phys. Rev. B 70, 174203 (2004).

[42] M. Leitner, W. Schroer, and G. Pottlacher, J. Thermophys. 39, 124 (2018).
[43] P. F. Paradis, T. Ishikawa, and S. Yoda, J. Appl. Phys. 97, 053506 (2005).

[44] E. N. Hahn, T. C. Germann, R. Ravelo, J. E. Hammerberg, and M. A. Meyers, Acta Mater. 126, 313 (2017).

[45] J. C. Fisher, J. Appl. Phys. 19, 1062 (1948).

[46] L. J. Briggs, J. Appl. Phys. 21, 721 (1950).

[47] S. I. Ashitkov, P. S. Komarov, E. V Struleva, M. B. Agranat, G. I. Kanel, and K. V. Khishchenko, J. Phys. Conf. Ser. 653, 012001 (2015).

[48] L. Cortella, B. Vinet, P. J. Desré, A. Pasturel, A. T. Paxton, and M. van Schilfgaarde, Phys. Rev. Lett. 70, 1469 (1993). 